\title{
COMPUTATIONS WITH MODIFIED DIAGONALS
}

\author{
KIERAN G. O'GRADY \\ "SAPIENZA"UNIVERSITÀ DI ROMA \\ Alla piccola Titti
}

\begin{abstract}
Motivated by conjectures of Beauville and Voisin on the Chow ring of Hyperkähler varieties we will prove some basic results on the rational equivalence class of modified diagonals of projective varieties.
\end{abstract}

Key Words: Chow ring, Hyperkähler varieties, modified diagonals.

Mathematics Subject Classification: 14C25, 14J28.

\section{Contents}

0. Introduction

1. Preliminaries

2. Products

3. $\mathbb{P}^{r}$-fibrations

4. Blow-ups

5. Double covers

References

\section{INTRODUCTION}

Let $X$ be an $n$-dimensional variety over a field $\mathbb{K}$ and $a \in X(\mathbb{K})$. For $I \subset\{1, \ldots, m\}$ we let

$$
\Delta_{I}^{m}(X ; a):=\left\{\left(x_{1}, \ldots, x_{m}\right) \in X^{m} \mid x_{i}=x_{j} \text { if } i, j \in I \text { and } x_{i}=a \text { if } i \notin I\right\} .
$$

The $m$-th modified diagonal cycle associated to $a$ is the $n$-cycle on $X^{m}$ given by

$$
\Gamma^{m}(X ; a):=\sum_{\emptyset \neq I \subset\{1,2, \ldots, m\}}(-1)^{m-|I|} \Delta_{I}^{m}(X ; a)
$$

if $n>0$, and equal to 0 if $n=0$. Gross and Schoen [6] proved that if $X$ is a (smooth projective) hyperelliptic curve and $a$ is a fixed point of a hyperelliptic involution then $\Gamma^{3}(X ; a)$ represents a torsion class in the Chow group of $X^{3}$. On the other hand it is known that if $X$ is a generic complex smooth plane curve and $m$ is small compared to its genus then $\Gamma^{m}(X ; a)$ is not algebraically equivalent to 0 , whatever $a$ is, see [11] (for the link between vanishing of $\Gamma^{m}(X ; a)$ and Voisin's result on the Beauville decomposition of the Abel-Jacobi image of a curve see the proof of Prop.4.3 of [3]). Let $X$ be a complex projective $K 3$ surface: Beauville and Voisin [3 have proved that there exists $c \in X$ such that the rational equivalence class of $\Gamma^{3}(X ; c)$ is torsion. A natural question arises: under which hypotheses a modified diagonal cycle on a projective variety represents a torsion class in the Chow group? We should point out that such a vanishing can entail unexpected geometric properties: if $X$ is a smooth projective variety of dimension $n$ and $\Gamma^{n+1}(X ; a)$ is torsion in the Chow group then the intersection of arbitrary divisor classes $D_{1}, \ldots, D_{n}$ on $X$ is rationally equivalent to a multiple of $a$. A set of conjectures put forth by Beauville [2] and Voisin [10] predict exactly such a degenerate behaviour for the intersection product of divisors on hyperkähler varieties i.e. complex smooth projective varieties which are simply connected and carry a holomorphic symplectic form whose cohomology class spans $H^{2,0}$ (see [7, 9] for more results on those conjectures). Our interest in modified diagonals has been motivated by the desire to prove the conjecture on hyperkähler varieties stated below. From now on

Date: March 232014.

Supported by PRIN 2010. 
the notation $A \equiv B$ for cycles $A, B$ on a variety $X$ means that for some integer $d \neq 0$ the cycle $d A$ is rationally equivalent to $d B$, i.e. we will work with the rational Chow group $\mathrm{CH}(X)_{\mathbb{Q}}:=\mathrm{CH}(X) \otimes_{\mathbb{Z}} \mathbb{Q}$.

Conjecture 0.1. Let $X$ be a Hyperkähler variety of dimension $2 n$. Then there exists a $\in X$ such that $\Gamma^{2 n+1}(X ; a) \equiv 0$.

In the present paper we will not prove Conjecture 0.1 instead we will establish a few basic results on modified diagonals. Below is our first result, see Section 2 .

Proposition 0.2. Let $X, Y$ be smooth projective varieties. Suppose that there exist $a \in X(\mathbb{K}), b \in Y(\mathbb{K})$ such that $\Gamma^{m}(X ; a) \equiv 0$ and $\Gamma^{n}(Y ; b) \equiv 0$. Then $\Gamma^{m+n-1}(X \times Y ;(a, b)) \equiv 0$.

We will apply the above proposition in order to show that if $T$ is a complex abelian surface and $a \in T$ then $\Gamma^{5}(T ; a) \equiv 0$. Notice that if $E$ is an elliptic curve and $a \in E$ then $\Gamma^{3}(E ; a) \equiv 0$ by Gross and Schoen [6]. These results are particular instances of a Theorem of Moonen and Yin [8] which asserts that $\Gamma^{2 g+1}(A ; p) \equiv 0$ for $A$ an abelian variety of dimension $g$ and $p \in A(\mathbb{K})$ (and more generally for an abelian scheme of relative dimension $g$ ). A word about the relation between Moonen - Yin's result and Conjecture 0.1. Beauville and Voisin proved that the relation $\Gamma^{3}(X ; c) \equiv 0$ for $X$ a complex projective $K 3$ surface (and a certain $c \in X$ ) follows from the existence of an elliptic surface $Y$ dominating $X$ and the relation $\Gamma^{3}\left(E_{t} ; a\right) \equiv 0$ for the fibers of the elliptic fibration on $Y$. We expect that the theorem of Moonen and Yin can be used to prove that Conjecture $\mathbf{0 . 1}$ holds for Hyperkähler varieties which are covered generically by abelian varieties, this is the subject of work in progress. (It is hard to believe that every Hyperkähler variety of dimension greater than 2 is covered generically by abelian varieties, but certainly there are interesting codimension- 1 families which have this property, viz. lagrangian fibrations and Hilbert schemes of $K 3$ surfaces, moreover Lang's conjectures on hyperbolicity would give that a hyperkähler variety is generically covered by varieties birational to abelian varieties.) In Section $\mathbf{3}$ we will prove that, in a certain sense, Proposition $\mathbf{0 . 2}$ holds also for $\mathbb{P}^{r}$ fibrations over smooth projective varieties if certain hypotheses are satisfied, then we will apply the result to prove vanishing of classes of modified diagonals of symmetric products of curves of genus at most 2. In Section 4 we will prove the following result.

Proposition 0.3. Let $Y$ be a smooth projective variety and $V \subset Y$ be a smooth subvariety of codimension e. Suppose that there exists $b \in V(\mathbb{K})$ such that $\Gamma^{n+1}(Y ; b) \equiv 0$ and $\Gamma^{n-e+1}(V ; b) \equiv 0$. Let $X \rightarrow Y$ be the blow-up of $V$ and $a \in X(\mathbb{K})$ such that $f(a)=b$. Then $\Gamma^{n+1}(X ; a) \equiv 0$.

We will apply Proposition $\mathbf{0 . 3}$ and Proposition $\mathbf{0 . 2}$ in order to show that Conjecture $\mathbf{0 . 1}$ holds for $S^{[n]}$ where $S$ is a complex $K 3$ surface and $n=2,3$, see Proposition 4.7. In Section [5] we will consider double covers $f: X \rightarrow Y$ where $X$ is a projective variety. We will prove that if $a \in X(\mathbb{K})$ is a ramification point and $\Gamma^{m}(Y ; f(a)) \equiv 0$ then $\Gamma^{2 m-1}(X ; a) \equiv 0$, provided $m=2,3$. The proof for $m=2$ is the proof, given by Gross and Schoen, that if $X$ is a hyperelliptic curve then $\Gamma^{3}(X ; a) \equiv 0$ for $a \in X(\mathbb{K})$ a fixed point of a hyperelliptic involution; we expect that our extension will work for arbitrary $m$ but we have not been able to carry out the necessary linear algebra computations. The result for $m=3$ allows us to give another proof that $\Gamma^{5}(T ; a) \equiv 0$ for a complex abelian surface $T$ : the equality $\Gamma^{5}(T ; a) \equiv 0$ follows from our result on double covers and the equality $\Gamma^{3}(T /\langle-1\rangle ; c) \equiv 0$ proved by Beauville and Voisin [3].

0.1. Conventions and notation. Varieties are defined over a base field $\mathbb{K}$. A point of $X$ is an element of $X(\mathbb{K})$. We denote the small diagonal $\Delta_{\{1, \ldots, m\}}^{m}(X ; a)$ by $\Delta^{m}(X)$ and we let $\pi_{i}^{m}: X^{m} \rightarrow X$ be the $i$-th projection - we will drop the superscript $m$ if there is no potential for confusion. We let $X^{(n)}$ be the $n$-th symmetric product of $X$ i.e. $X^{(n)}:=X^{n} / \mathcal{S}_{n}$ where $\mathcal{S}_{n}$ is the symmetric group on $n$ elements.

0.2. Acknowledgments. It is a pleasure to thank Lie Fu, Ben Moonen and Charles Vial for the interest they took in this work.

\section{Preliminaries}

1.1. Let $X$ be an $n$-dimensional projective variety over a field $\mathbb{K}, a \in X(\mathbb{K})$ and $h$ a hyperplane class on $X$. Let $\iota: \Delta^{m}(X) \hookrightarrow X^{m}$ be the inclusion map. If $m \leq n$ then

$$
\Gamma^{m}(X ; a) \cdot \pi_{1}^{*}(h) \cdot \pi_{2}^{*}(h) \cdot \ldots \cdot \pi_{m-1}^{*}(h) \cdot \pi_{m}\left(h^{n-m+1}\right)=\iota_{*}\left(h^{n}\right) .
$$


Since $\operatorname{deg} \iota_{*}\left(h^{n}\right) \neq 0$ it follows that $\Gamma^{m}(X ; a) \not \equiv 0$ if $m \leq n$. Now suppose that $\Gamma^{n+1}(X ; a) \equiv 0$. Let $D_{1}, \ldots, D_{n}$ be Cartier divisors on $X$ : then

$$
0=\pi_{n+1, *}\left(\Gamma^{n+1}(X ; a) \cdot \pi_{1}^{*} D_{1} \cdot \ldots \cdot \pi_{n}^{*} D_{n}\right)=D_{1} \cdot \ldots \cdot D_{n}-\operatorname{deg}\left(D_{1} \cdot \ldots \cdot D_{n}\right) a
$$

in $\mathrm{CH}_{0}(X)_{\mathbb{Q}}$.

Remark 1.1. Equation (1.1.2) shows that if $\Gamma^{n+1}(X ; a) \equiv 0$ and $\Gamma^{n+1}(X ; b) \equiv 0$ then $a \equiv b$.

Example 1.2. The intersection product between cycle classes of complementary dimension defines a perfect pairing on $\mathrm{CH}\left(\left(\mathbb{P}^{n}\right)^{m}\right)$. Let $a \in \mathbb{P}^{n}$ : since $\Gamma^{n+1}\left(\mathbb{P}^{n} ; a\right)$ pairs to 0 with any class of complementary dimension it follows that $\Gamma^{n+1}\left(\mathbb{P}^{n} ; a\right) \equiv 0$.

1.2. In the present subsection we will assume that $X$ is a complex smooth projective variety of dimension $n$. Let $a \in X$. Let $\alpha_{1}, \ldots, \alpha_{m} \in H_{D R}(X)$ be De Rham homogeneous cohomology classes such that $\sum_{i=1}^{m} \operatorname{deg} \alpha_{i}=2 n$. Thus it makes sense to integrate $\pi_{1}^{*} \alpha_{1} \wedge \ldots \wedge \pi_{m}^{*} \alpha_{m}$ on $\Gamma^{m}(X ; a)$. Let

$$
s:=\left|\left\{1 \leq i \leq m \mid \operatorname{deg} \alpha_{i}=0\right\}\right| .
$$

A straightforward computation gives that

$$
\int_{\Gamma^{m}(X ; a)} \pi_{1}^{*} \alpha_{1} \wedge \ldots \wedge \pi_{m}^{*} \alpha_{m}=\sum_{\ell=0}^{m-1}(-1)^{\ell}\left(\begin{array}{l}
s \\
\ell
\end{array}\right) \int_{X} \alpha_{1} \wedge \ldots \wedge \alpha_{m} .
$$

Proposition 1.3. Let $X$ be a smooth complex projective variety and $a \in X$. Let $n$ be the dimension of $X$ and $d$ be its Albanese dimension. The homology class of $\Gamma^{m}(X ; a)$ is torsion if and only if $m>(n+d)$.

Proof. If $n=0$ the result is obvious. From now on we assume that $n>0$. By (1.1.1) we may assume that $m>n$. The homology class of $\Gamma^{m}(X ; a)$ is torsion if and only if the left-hand side of (1.2.2) vanishes for every choice of homogeneous $\alpha_{1}, \ldots, \alpha_{m} \in H_{D R}(X)$ such that $\sum_{i=1}^{m} \operatorname{deg} \alpha_{i}=2 n$. Suppose first that $n<m \leq(n+d)$ and let $m=n+e$ : thus $0<e \leq d$. Choose a point of $X$ and let $\operatorname{alb}_{X}: X \rightarrow \operatorname{Alb}(X)$ be the associated Albanese map. Let $\theta$ be a a Kähler form on $\operatorname{Alb}(X)$ : by hypothesis $\operatorname{dim}\left(\operatorname{Im} \operatorname{alb}_{X}\right)=d$ and hence there exist holomorphic 1 -forms $\psi_{1}, \ldots, \psi_{e}$ on $\operatorname{Alb}(X)$ such that

$$
\int_{\operatorname{Im}\left(\operatorname{alb}_{X}\right)} \psi_{1} \wedge \ldots \wedge \psi_{e} \wedge \bar{\psi}_{1} \wedge \ldots \wedge \bar{\psi}_{e} \wedge \theta^{d-e}>0
$$

For $i=1, \ldots, e$ let $\phi_{i}:=\operatorname{alb}_{X}^{*} \psi_{i}$ and $\eta:=\operatorname{alb}_{X}^{*} \theta$. Let $\omega \in H_{D R}^{2}(X)$ be a Kähler class. Equations (1.2.2) and (1.2.3) give that

$$
\int_{\Gamma^{m}(X ; a)} \pi_{1}^{*} \phi_{1} \wedge \ldots \wedge \pi_{e}^{*} \phi_{e} \wedge \pi_{e+1}^{*} \bar{\phi}_{1} \wedge \ldots \wedge \pi_{2 e}^{*} \bar{\phi}_{e} \wedge \pi_{2 e+1}^{*} \eta \wedge \ldots \wedge \pi_{e+d}^{*} \eta \wedge \pi_{e+d+1}^{*} \omega \wedge \ldots \wedge \pi_{m}^{*} \omega=\int_{X} \phi_{1} \wedge \ldots \wedge \phi_{e} \wedge \bar{\phi}_{1} \wedge \ldots \wedge \bar{\phi}_{e} \wedge \eta^{d-e} \wedge \omega^{n-d}>0
$$

It follows that the homology class of $\Gamma^{m}(X ; a)$ is not torsion. Lastly suppose that $m>(n+d)$. Let $s$ be given by (1.2.1): then $s \leq(m-1)$ because $n>0$. It follows that if $s>0$ the right-hand side of (1.2.2) vanishes (by the binomial formula). Now assume that $s=0$ : by (1.2.2) we have that

$$
\int_{\Gamma^{m}(X ; a)} \pi_{1}^{*} \alpha_{1} \wedge \ldots \wedge \pi_{m}^{*} \alpha_{m}=\int_{X} \alpha_{1} \wedge \ldots \wedge \alpha_{m}
$$

Let

$$
t:=\left|\left\{1 \leq i \leq m \mid \operatorname{deg} \alpha_{i}=1\right\}\right| .
$$

If $t>2 d$ then the right-hand side of (1.2.5) vanishes because every class in $H_{D R}^{1}(X)$ is represented by the pull-back of a closed 1-form on $\operatorname{Alb}(X)$ via the Albanese map and by hypothesis $\operatorname{dim}\left(\operatorname{Im} \operatorname{alb}_{X}\right)=d$. Now suppose that $t \leq 2 d$. Then

$$
\operatorname{deg}\left(\pi_{1}^{*} \alpha_{1} \wedge \ldots \wedge \pi_{m}^{*} \alpha_{m}\right) \geq t+2(m-t)>2 n+2 d-t \geq 2 n
$$

and hence the right-hand side of (1.2.5) vanishes because the integrand is identically zero. This proves that if $m>(n+d)$ the homology class of $\Gamma^{m}(X ; a)$ is torsion.

1.3. Let $f: X \rightarrow Y$ be a map of finite non-zero degree between projective varieties. Let $a \in X$ and $b:=f(a)$. Then $f_{*} \Gamma^{m}(X ; a)=(\operatorname{deg} f) \Gamma^{m}(Y ; b)$. It follows that if $\Gamma^{m}(X ; a) \equiv 0$ then $\Gamma^{m}(Y ; b) \equiv 0$. 


\section{Products}

We will prove Proposition $\mathbf{0 . 2}$ and then we will prove that if $T$ is a complex abelian surface then $\Gamma^{5}(T ; a) \equiv 0$ for any $a \in T$.

2.1. Preliminary computations. Let $X$ and $Y$ be projective varieties and $a \in X, b \in Y$. Let $\emptyset \neq I \subset\{1, \ldots, r\}$ and $\emptyset \neq J \subset\{1, \ldots, s\}$. Thus $\Delta_{I}^{r}(X ; a) \subset X^{r}$ and $\Delta_{J}^{s}(Y ; b) \subset Y^{s}$ : we let

$$
\Delta_{I, J}^{r, s}(X, Y ; a, b):=\Delta_{I}^{r}(X ; a) \times \Delta_{J}^{s}(Y ; b) \subset X^{r} \times Y^{s} .
$$

We let $\Delta^{r, s}(X, Y)=\Delta_{\{1, \ldots, r\},\{1, \ldots, s\}}^{r, s}(X, Y ; a, b)$. For the remainder of the present section we let

$$
e:=m+n-1 \text {. }
$$

We will constantly make the identification

$$
\begin{array}{ccc}
(X \times Y)^{e} & \stackrel{\sim}{\longrightarrow} & X^{e} \times Y^{e} \\
\left(\left(x_{1}, y_{1}\right), \ldots,\left(x_{e}, y_{e}\right)\right) & \mapsto & \left(x_{1}, \ldots, x_{e}, y_{1}, \ldots, y_{e}\right)
\end{array}
$$

With the above notation Proposition $\mathbf{0 . 2}$ is equivalent to the following rational equivalence:

$$
\sum_{\emptyset \neq I \subset\{1, \ldots, e\}}(-1)^{e-|I|} \Delta_{I, I}^{e, e}(X, Y ; a, b) \equiv 0 .
$$

Proposition 2.1. Let $X$ be a smooth projective variety and $a \in X$. Suppose that $\Gamma^{m}(X ; a) \equiv 0$. Then

$$
\Delta^{m+r}(X) \equiv \sum_{1 \leq|J| \leq(m-1)}(-1)^{m-1-|J|}\left(\begin{array}{c}
m+r-1-|J| \\
r
\end{array}\right) \Delta_{J}^{m+r}(X ; a)
$$

for every $r \geq 0$.

Proof. By induction on $r$. If $r=0$ then (2.1.5) is equivalent to $\Gamma^{m}(X ; a) \equiv 0$. Let's prove the inductive step. Since $\Gamma^{m}(X ; a) \equiv 0$ we have that

$$
\begin{aligned}
& \Delta^{m+r+1}(X) \equiv \pi_{1, \ldots, m+r}^{*} \Delta^{m+r}(X) \cdot \pi_{m+r, m+r+1}^{*} \Delta^{2}(X) \equiv \\
\equiv & \pi_{1, \ldots, m+r}^{*}\left(\sum_{\substack{J \subset\{1, \ldots, m+r\} \\
1 \leq|J| \leq(m-1)}}(-1)^{m-1-|J|}\left(\begin{array}{c}
m+r-1-|J| \\
r
\end{array}\right) \Delta_{J}^{m+r}(X ; a)\right) \cdot \pi_{m+r, m+r+1}^{*} \Delta^{2}(X) .
\end{aligned}
$$

Next notice that

$$
\pi_{1, \ldots, m+r}^{*} \Delta_{J}^{m+r}(X ; a) \cdot \pi_{m+r, m+r+1}^{*} \Delta^{2}(X) \equiv \begin{cases}\Delta_{J}^{m+r+1}(X ; a) & \text { if }(m+r) \notin J, \\ \Delta_{J \cup\{m+r+1\}}^{m+r+1}(X ; a) & \text { if }(m+r) \in J,\end{cases}
$$

Thus $\Delta^{m+r+1}(X)$ is rationally equivalent to a linear combination of cycles $\Delta_{J}^{m+r+1}(X ; a)$ with $|J| \leq$ $(m-1)$ and of cycles $\Delta_{K}^{m+r+1}(X ; a)$ where

$$
|K|=m, \quad\{m+r, m+r+1\} \subset K .
$$

Let $K$ be such a subset and write $K=\left\{i_{1}, \ldots, i_{m}\right\}$ where $i_{1}<\ldots<i_{m}$. Let $\iota: X^{m} \rightarrow X^{m+r+1}$ be the map which composed with the $j$-th projection of $X^{m+r+1}$ is equal to the constant map to $a$ if $j \notin K$, and is equal to the $l$-th projection of $X^{m}$ if if $j=i_{l}$. Then $\Delta_{K}^{m+r+1}(X ; a)=\iota_{*} \Delta^{m}$ and hence the equivalence $\Gamma^{m}(a) \equiv 0$ gives that

$$
\Delta_{K}^{m+r+1}(X ; a) \equiv \sum_{\substack{J \subset K \\ 1 \leq|J| \leq(m-1)}}(-1)^{m-1-|J|} \Delta_{J}^{m+r+1}(X ; a) .
$$

Putting everything together we get an equivalence

$$
\Delta^{m+r+1}(X) \equiv \sum_{1 \leq|J| \leq(m-1)}(-1)^{m-1-|J|} c_{J} \Delta_{J}^{m+r+1}(X ; a)
$$

In order to prove that $c_{J}=\left(\begin{array}{c}m+r-|J| \\ r+1\end{array}\right)$ we distinguish four cases: they are indexed by the intersection

$$
J \cap\{m+r, m+r+1\} .
$$

Suppose that (2.1.11) is empty. We get a contribution (to $\left.c_{J}\right)$ of $\left(\begin{array}{c}m+r-1-|J| \\ r\end{array}\right)$ from the first case in (2.1.7), and a contribution of

$$
|\{(J \cup\{m+r, m+r+1\}) \subset K \subset\{1, \ldots, m+r+1\}|| K \mid=m\}|=\left(\begin{array}{c}
m+r-1-|J| \\
m-2-|J|
\end{array}\right)=\left(\begin{array}{c}
m+r-1-|J| \\
r+1
\end{array}\right)
$$


from the subsets $K$ satisfying (2.1.8). This proves that $c_{J}=\left(\begin{array}{c}m+r-|J| \\ r+1\end{array}\right)$ in this case. The proof in the other three cases is similar.

Corollary 2.2. Let $X$ be a smooth projective variety and $a \in X$. Suppose that $\Gamma^{m}(X ; a) \equiv 0$. Let $s \geq 0$ and $I \subset\{1, \ldots, m+s\}$ be a subset of cardinality at least $m$. Then

$$
\Delta_{I}^{m+s}(X ; a) \equiv \sum_{\substack{J \subset I \\
1 \leq|J| \leq(m-1)}}(-1)^{m-1-|J|}\left(\begin{array}{c}
|I|-|J|-1 \\
|I|-m
\end{array}\right) \Delta_{J}^{m+s}(X ; a) .
$$

Proof. Let $q:=|I|$ and $I=\left\{i_{1}, \ldots, i_{q}\right\}$ where $i_{1}<\ldots<i_{q}$. Let $\iota: X^{q} \rightarrow X^{m+s}$ be the map which composed with the $j$-th projection of $X^{m+s}$ is equal to the constant map to $a$ if $j \notin I$, and is equal to the $l$-th projection of $X^{m}$ if if $j=i_{l}$. Then $\Delta_{I}^{m+s}(X ; a)=\iota_{*} \Delta^{q}(X)$ and one gets (2.1.13) by invoking Proposition 2.1.

Corollary 2.3. Let $X, Y$ be smooth projective varieties and $a \in X, b \in Y$. Suppose that $\Gamma^{m}(X ; a) \equiv 0$ and $\Gamma^{n}(Y ; a) \equiv 0$. Assume that $m \leq n$. Let $I \subset\{1, \ldots, e\}$ (recall that $\left.e=m+n-1\right)$.

(1) If $n \leq|I|$ then

$$
\Delta_{I, I}^{e, e}(X, Y ; a, b) \equiv \sum_{\substack{(J \cup K) \subset I \\
1 \leq|J| \leq(m-1) \\
1 \leq|K| \leq(n-1)}}(-1)^{m+n-|J|-|K|}\left(\begin{array}{c}
|I|-|J|-1 \\
m-|J|-1
\end{array}\right)\left(\begin{array}{c}
|I|-|K|-1 \\
n-|K|-1
\end{array}\right) \Delta_{J, K}^{e, e}(X, Y ; a, b) .
$$

(2) If $m \leq|I|<n$ then

$$
\Delta_{I, I}^{e, e}(X, Y ; a, b) \equiv \sum_{\substack{J \subset I \\
1 \leq|J| \leq(m-1)}}(-1)^{m-1-|J|}\left(\begin{array}{c}
|I|-|J|-1 \\
m-|J|-1
\end{array}\right) \Delta_{J, I}^{e, e}(X, Y ; a, b) .
$$

Proof. By definition $\Delta_{I, I}^{e, e}(X, Y ; a, b)=\Delta_{I}^{e}(X ; a) \times \Delta_{I}^{e}(Y ; b)$. Now suppose that $n \leq|I|$. By Corollary 2.2 the first factor is rationally equivalent to a linear combination of $\Delta_{J}^{e}(X ; a)$ 's with $J \subset I$ and $1 \leq|J| \leq(m-1)$, the second factor is rationally equivalent to a linear combination of $\Delta_{K}^{e}(Y ; b)$ 's with $K \subset I$ and $1 \leq|K| \leq(n-1)$ : writing out the product one gets (2.1.14). The proof of (2.1.15) is similar.

2.2. Linear relations between binomial coefficients. The following fact will be useful:

$$
\sum_{t=0}^{n}(-1)^{t} p(t)\left(\begin{array}{l}
n \\
t
\end{array}\right)=0 \quad \forall p \in \mathbb{Q}[x] \text { such that } \operatorname{deg} p<n .
$$

In order to prove (2.2.1) let $d<n$ : then we have

$$
\sum_{t=0}^{n}(-1)^{t}\left(\begin{array}{l}
t \\
d
\end{array}\right)\left(\begin{array}{l}
n \\
t
\end{array}\right)=\left(\begin{array}{l}
n \\
d
\end{array}\right) \sum_{t=d}^{n}(-1)^{t}\left(\begin{array}{l}
n-d \\
t-d
\end{array}\right)=(1-1)^{n-d}=0 .
$$

Since $\left\{\left(\begin{array}{l}x \\ 0\end{array}\right),\left(\begin{array}{l}x \\ 1\end{array}\right), \ldots,\left(\begin{array}{c}x \\ n-1\end{array}\right)\right\}$ is a basis of the vector space of polynomials of degree at most $(n-1)$ Equation (2.2.1) follows.

2.3. Proof of the main result. We will prove Proposition $\mathbf{0 . 2}$ As noticed above it suffices to prove that (2.1.4) holds. Without loss of generality we may assume that $m \leq n$. Corollary 2.3 gives that for each $1 \leq t \leq e$ and $J, K \subset\{1, \ldots, e\}$ with $|J| \leq(m-1),|K| \leq(n-1)$ there exists $c_{J, K}(t)$ such that

$$
\sum_{|I|=t} \Delta_{I, I}^{e, e}(X, Y ; a, b) \equiv \sum_{\substack{J, K \subset\{1, \ldots, e\} \\ 1 \leq|J| \leq(m-1) \\ 1 \leq|K| \leq(n-1)}} c_{J, K}(t) \Delta_{J, K}^{e, e}(X, Y ; a, b)
$$

It will suffice to prove that for each $J, K$ as above we have

$$
\sum_{t=1}^{e}(-1)^{t} c_{J, K}(t)=0 \text {. }
$$

Equations (2.1.14) and (2.1.15) give that $c_{J, K}(t)=0$ if $t<|J \cup K|$ and that

$$
c_{J, K}(t)=(-1)^{m+n-|J|-|K|}\left(\begin{array}{c}
t-|J|-1 \\
m-|J|-1
\end{array}\right)\left(\begin{array}{c}
t-|K|-1 \\
n-|K|-1
\end{array}\right)\left(\begin{array}{c}
e-|J \cup K| \\
t-|J \cup K|
\end{array}\right), \quad \max \{|J \cup K|, n\} \leq t \leq e .
$$


We distinguish between the four cases:

(1) $J \not \subset K$.

(2) $J \subset K$ and $m \leq|K|$.

(3) $J \subset K, J \neq K$ and $|K|<m$.

(4) $J=K$ and $|K|<m$.

Suppose that (1) holds. Then Corollary 2.3 gives that $c_{J, K}(t)=0$ if $t<n$. Let $p \in \mathbb{Q}[x]$ be given by

$$
p:=(-1)^{m+n-|J|-|K|}\left(\begin{array}{c}
x-|J|-1 \\
m-|J|-1
\end{array}\right)\left(\begin{array}{c}
x-|K|-1 \\
n-|K|-1
\end{array}\right) .
$$

We must prove that

$$
\sum_{t=\max \{|J \cup K|, n\}}^{e}(-1)^{t} p(t)\left(\begin{array}{l}
e-|J \cup K| \\
t-|J \cup K|
\end{array}\right)=0 .
$$

If $n \leq|J \cup K|$ then (2.3.5) follows at once from (2.2.1) (notice that $\operatorname{deg} p<(e-|J \cup K|)$ ), if $n<|J \cup K|$ then (2.3.5) follows from (2.2.1) and the fact that $p(i)=0$ for $|J \cup K| \leq i \leq(n-1)$. This proves (2.3.2) if Item (1) above holds. Now let's assume that Item (2) above holds. Then $|J \cup K|=|K|<n$ : it follows that if $n \leq t$ then $c_{J, K}(t)$ is given by (2.3.3). On the other hand Corollary 2.3 gives that if $t<n$ and $t \neq|K|$ then $c_{J, K}(t)=0$, and

$$
c_{J, K}(|K|)=(-1)^{m-1-|J|}\left(\begin{array}{c}
|K|-|J|-1 \\
m-|J|-1
\end{array}\right) .
$$

Thus we must prove that

$$
(-1)^{|K|}(-1)^{m-1-|J|}\left(\begin{array}{c}
|K|-|J|-1 \\
m-|J|-1
\end{array}\right)+\sum_{t=n}^{e}(-1)^{t} p(t)\left(\begin{array}{c}
e-|J \cup K| \\
t-|J \cup K|
\end{array}\right)=0
$$

where $p$ is given by (2.3.4). Now notice that $0=p(|K|+1)=\ldots=p(n-1)$ : thus (2.2.1) gives that

$$
\sum_{t=n}^{e}(-1)^{t} p(t)\left(\begin{array}{c}
e-|J \cup K| \\
t-|J \cup K|
\end{array}\right)=-(-1)^{|K|} p(|K|)\left(\begin{array}{c}
e-|K| \\
0
\end{array}\right)=(-1)^{m+n-1-|J|}\left(\begin{array}{c}
|K|-|J|-1 \\
m-|J|-1
\end{array}\right)\left(\begin{array}{c}
-1 \\
n-|K|-1
\end{array}\right)=(-1)^{m-|J|-|K|}\left(\begin{array}{c}
|K|-|J|-1 \\
m-|J|-1
\end{array}\right) .
$$

This proves that (2.3.7) holds. If Item (3) above holds one proves (2.3.2) arguing as in Item (1), if Item (4) holds the argument is similar to that given if Item (2) holds.

2.4. Stability. We will prove a result that will be useful later on.

Proposition 2.4. Let $X$ be a smooth projective variety and $a \in X$. Suppose that $\Gamma^{m}(X ; a) \equiv 0$. If $s \geq 0$ then $\Gamma^{m+s}(X ; a) \equiv 0$.

Proof. If $\operatorname{dim} X=0$ the result is trivial. Assume that $\operatorname{dim} X>0$. By definition

$$
\Gamma^{m+s}(X ; a):=\sum_{\emptyset \neq I \subset\{1,2, \ldots, m+s\}}(-1)^{m+s-|I|} \Delta_{I}^{m+s}(X ; a) .
$$

Replacing $\Delta_{I}^{m+s}(X ; a)$ for $m \leq|I| \leq(m+s)$ by the right-hand side of (2.1.13) we get that

$$
\Gamma^{m+s}(X ; a):=\sum_{1 \leq \ell \leq(m-1)} c_{\ell}\left(\sum_{|I|=\ell}(-1) \Delta_{I}^{m+s}(X ; a)\right)
$$

where

$$
c_{\ell}=\sum_{r=0}^{s}(-1)^{m-\ell-1+s-r}\left(\begin{array}{c}
m-\ell-1+r \\
m-\ell-1
\end{array}\right)\left(\begin{array}{c}
m+s-\ell \\
s-r
\end{array}\right)+(-1)^{m+s-\ell} .
$$

Thus it suffices to prove that $c_{\ell}=0$ for $1 \leq \ell \leq(m-1)$. Letting $t=s-r$ we get that

$$
\begin{aligned}
(-1)^{m-\ell-1} c_{\ell}=\sum_{t=0}^{s}(-1)^{t}\left(\begin{array}{r}
m-\ell-1+s-t \\
m-\ell-1
\end{array}\right)\left(\begin{array}{c}
m+s-\ell \\
t
\end{array}\right)+(-1)^{s-1}= \\
\quad=\sum_{t=0}^{m+s-\ell}(-1)^{t}\left(\begin{array}{c}
m-\ell-1+s-t \\
m-\ell-1
\end{array}\right)\left(\begin{array}{c}
m+s-\ell \\
t
\end{array}\right)=0
\end{aligned}
$$

where the last equality follows from (2.2.1). 


\subsection{Applications.}

Proposition 2.5. Suppose that $C$ is a smooth projective curve of genus $g$ and that there exists a degree-2 map $f: C \rightarrow \mathbb{P}^{1}$ ramified at $p \in C$. Then

(1) $\Gamma^{2 g+1}\left(C^{g} ;(p, \ldots, p)\right) \equiv 0$,

(2) $\Gamma^{2 g+1}\left(C^{(g)} ; g p\right) \equiv 0$, and

(3) $\Gamma^{2 g+1}\left(\operatorname{Pic}^{0}(C) ; a\right) \equiv 0$ for any $a \in \operatorname{Pic}^{0}(C)$.

Proof. By Proposition 4.8 of [6] we have $\Gamma^{3}(C ; p) \equiv 0$. Repeated application of Proposition $\mathbf{0 . 2}$ gives the first item. The quotient map $C^{g} \rightarrow C^{(g)}$ is finite and the image of $(p, \ldots, p)$ is gp: thus Item (2) follows from Item (1) and Subsection 1.3. Let $u_{g}: C^{(g)} \rightarrow \operatorname{Pic}^{0}(C)$ be the map $D \mapsto[D-g p]$ : since $u_{g}$ is birational Item $(2)$ and Subsection $\mathbf{1 . 3}$ give that $\Gamma^{2 g+1}\left(\operatorname{Pic}^{0}(C) ; \mathbf{0}\right) \equiv 0$ where $\mathbf{0}$ is the origin of $\operatorname{Pic}^{0}(C)$. Acting by translations we get that $\Gamma^{2 g+1}\left(\operatorname{Pic}^{0}(C) ; a\right) \equiv 0$ for any $a \in \operatorname{Pic}^{0}(C)$.

Corollary 2.6. If $T$ is a complex abelian surface then $\Gamma^{5}(T ; a) \equiv 0$ for any $a \in T$.

Proof. There exists a principally polarized abelian surface $J$ and an isogeny $J \rightarrow T$. By Subsection 1.3 it suffices to prove that $\Gamma^{5}(J ; b) \equiv 0$ for any $b \in J$. The surface $J$ is either a product of two elliptic curves $E_{1}, E_{2}$ or the Jacobian of a smooth genus-2 curve $C$. Suppose that the former holds. Let $a=\left(p_{1}, p_{2}\right)$ where $p_{i} \in E_{i}$ for $i=1,2$. Then $\Gamma^{3}\left(E_{i} ; p_{i}\right) \equiv 0$ by Proposition 4.8 of $[6$ and hence Proposition $\mathbf{0 . 2}$ gives that $\Gamma^{5}\left(E_{1} \times E_{2} ;\left(p_{1}, p_{2}\right)\right) \equiv 0$. If $J$ is the Jacobian of a smooth genus-2 curve $C$ the corollary follows at once from Proposition 2.5

\section{3. $\mathbb{P}^{r}$-FIBRATIONS}

Let $Y$ be a smooth projective variety. Let $\mathscr{F}$ be a locally-free sheaf of rank $(r+1)$ on $Y$ and $X:=\mathbb{P}(\mathscr{F})$. Thus the structure map $\rho: X \rightarrow Y$ is a $\mathbb{P}^{r}$-fibration. Let $Z:=c_{1}\left(\mathscr{O}_{X}(1)\right) \in \mathrm{CH}^{1}(X)$. Suppose that there exists $b \in Y$ such that $\Gamma^{m}(Y ; b) \equiv 0$ and let $a \in \rho^{-1}(b)$. If $\mathbb{P}(\mathscr{F})$ is trivial then

$$
\Gamma^{m+r}(X ; a) \equiv 0
$$

by Example 1.2 and Proposition 0.2. In general (3.0.1) does not hold. In fact suppose that $Y$ is a $K 3$ surface and hence $\Gamma^{3}(Y ; b) \equiv 0$ where $b$ is a point lying on a rational curve [3]. If $\Gamma^{3+r}(X ; a) \equiv 0$ then the top self-intersection of any divisor class on $X$ is a multiple of $[a]$, see Subsection [1.1 considering $Z^{r+2}$ we get that $c_{2}(\mathscr{F})$ is a multiple of $[b]$. We will prove the following results.

Proposition 3.1. Keep notation as above and suppose that $\operatorname{dim} Y=1$. If $\Gamma^{m}(Y ; b) \equiv 0$ then $\Gamma^{m+r}(X ; a) \equiv 0$.

Proposition 3.2. Keep notation as above and suppose that $\operatorname{dim} Y=2$. If $\Gamma^{m-1}(Y ; b) \equiv 0$, or $\Gamma^{m}(Y ; b) \equiv 0$ and both $c_{1}(\mathscr{F})^{2}, c_{2}(\mathscr{F})$ are multiples of $[b]$, then $\Gamma^{m+r}(X ; a) \equiv 0$.

As an appplication we will prove the following.

Proposition 3.3. Suppose that $C$ is a smooth projective curve of genus $g \leq 2$ over an algebraically closed field $\mathbb{K}$ and that $p \in C$ is such that $\operatorname{dim}\left|\mathscr{O}_{C}(2 p)\right| \geq 1$. Then $\Gamma^{d+g+1}\left(C^{(d)} ; d p\right) \equiv 0$ for any $d \geq 0$.

3.1. Comparing diagonals. Let $\rho^{n}: X^{n} \rightarrow Y^{n}$ be the $n$-th cartesian product of $\rho$. Let $\pi_{i}: X^{n} \rightarrow X$ be the $i$-th projection and $Z_{i}:=\pi_{i}^{*} Z$. Given a multi-index $E=\left(e_{1}, \ldots, e_{n}\right)$ with $0 \leq e_{i}$ for $1 \leq i \leq n$ we let $Z^{E}:=Z_{1}^{e_{1}} \cdot \ldots \cdot Z_{n}^{e_{n}}$. We let

$$
\max E:=\max \left\{e_{1}, \ldots, e_{n}\right\}, \quad|E|:=e_{1}+\ldots+e_{n} .
$$

Let $d:=\operatorname{dim} Y$ and $\left[\Delta^{n}(X)\right] \in \mathrm{CH}_{d+r}\left(X^{n}\right)$ be the class of the (smallest) diagonal. Since $\rho^{n}$ is a $\left(\mathbb{P}^{r}\right)^{n}$-fibration we may write

$$
\left[\Delta^{n}(X)\right]=\sum_{\max E \leq r}\left(\rho^{n}\right)^{*}\left(w_{E}(\mathscr{F})\right) \cdot Z^{E}, \quad w_{E}(\mathscr{F}) \in \mathrm{CH}_{|E|+d-r(n-1)}\left(Y^{n}\right) .
$$

In order to describe the classes $w_{E}$ we let $\delta_{Y}^{n}: Y \hookrightarrow Y^{n}$ and $\delta_{X}^{n}: X \hookrightarrow X^{n}$ be the diagonal embeddings.

Proposition 3.4. Let $r \geq 0$ and $E=\left(e_{1}, \ldots, e_{n}\right)$ be a multi-index. There exists a universal polynomial $P_{E} \in \mathbb{Q}\left[x_{1}, \ldots, x_{q}\right]$, where $q:=(r(n-1)-|E|)$, such that the following holds. Let $\mathscr{F}$ be a locally-free sheaf of rank $(r+1)$ on $Y$ : then (notation as above) $w_{E}(\mathscr{F})=\delta_{Y, *}^{n}\left(P_{E}\left(c_{1}(\mathscr{F}), \ldots, c_{q}(\mathscr{F})\right)\right.$. 
Proof. Let $s_{i}(\mathscr{F})$ be the $i$-th Segre class of $\mathscr{F}$ and $E^{\vee}:=\left(r-e_{1}, \ldots, r-e_{n}\right)$. Then

$$
\rho_{*}^{n}\left(\left[\Delta^{n}(X)\right] \cdot Z^{E^{\vee}}\right)=\delta_{Y, *}^{n}\left(s_{\left|E^{\vee}\right|-r}(\mathscr{F})\right) .
$$

(By convention $s_{i}(\mathscr{F})=0$ if $i<0$.) On the other hand let $J=\left(j_{1}, \ldots, j_{n}\right)$ be a multi-index: then

$$
\rho_{*}^{n}\left(\left(\sum_{\max H \leq r}\left(\rho^{n}\right)^{*}\left(w_{H}(\mathscr{F})\right) \cdot Z^{H}\right) \cdot Z^{J}\right)=\sum_{\max H \leq r} w_{H}(\mathscr{F}) \cdot \pi_{1}^{*}\left(s_{h_{1}+j_{1}-r}\right) \cdot \ldots \cdot \pi_{n}^{*}\left(s_{h_{n}+j_{n}-r}\right) .
$$

Equations (3.1.3) and (3.1.4) give that

$$
\begin{aligned}
\delta_{Y, *}^{n}\left(s_{\left|E^{\vee}\right|-r}(\mathscr{F})\right)=\rho_{*}^{n}\left(\left[\Delta^{n}(X)\right] \cdot Z^{E^{\vee}}\right)=\sum_{\max H \leq r} w_{H}(\mathscr{F}) \cdot \pi_{1}^{*}\left(s_{h_{1}-e_{1}}\right) \cdot \ldots \cdot \pi_{n}^{*}\left(s_{h_{n}-e_{n}}\right)= \\
=w_{E}(\mathscr{F})+\sum_{\substack{|H|>|E| \\
r \geq \max H}} w_{H}(\mathscr{F}) \cdot \pi_{1}^{*}\left(s_{h_{1}-e_{1}}\right) \cdot \ldots \cdot \pi_{n}^{*}\left(s_{h_{n}-e_{n}}\right) .
\end{aligned}
$$

Starting from the highest possible value of $|E|$ i.e. $r n$ and going through descending values of $|E|$ one gets the proposition.

Remark 3.5. The proof of Proposition 3.4 gives an iterative algorithm for the computation of $w_{E}(\mathscr{F})$. A straightforward computation gives the formulae

$$
w_{E}(\mathscr{F})= \begin{cases}0 & \text { if }|E|>r(n-1), \\ {\left[\Delta^{n}(Y)\right]} & \text { if }|E|=r(n-1), \\ \left(\lambda_{E}(1)-1\right) \delta_{Y, *}^{n}\left(c_{1}(\mathscr{F})\right) & \text { if }|E|=r(n-1)-1, \\ \frac{1}{2}\left(\lambda_{E}(1)-1\right)\left(\lambda_{E}(1)-2\right) \delta_{Y, *}^{n}\left(c_{1}(\mathscr{F})^{2}\right)+\left(\lambda_{E}(2)-1\right) \delta_{Y, *}^{n}\left(c_{2}(\mathscr{F})\right) & \text { if }|E|=r(n-1)-2,\end{cases}
$$

where

$$
\lambda_{E}(p):=\left|\left\{1 \leq i \leq n \mid e_{i}+p \leq r\right\}\right|
$$

3.2. Comparing modified diagonals. We will compare $\Gamma^{m+r}(X ; a)$ and $\Gamma^{m+r}(Y ; b)$. In the present subsection $\emptyset \neq I \subset\{1, \ldots, m+r\}$ and $I^{c}:=(\{1, \ldots, m+r\} \backslash I)$; we let $\pi_{I}: X^{m+r} \rightarrow X^{|I|}$ be the projection determined by $I$. We also let $H=\left(h_{1}, \ldots, h_{m+r}\right)$ be a multi-index. If $\max H \leq r$ we let Top $H:=\left\{1 \leq i \leq n \mid h_{i}=r\right\}$. Applying Proposition 3.4 and Remark 3.5 we get that

$$
\begin{aligned}
& \Delta_{I}^{m+r}(X ; a)=\left(\rho^{m+r}\right)^{*}\left(\Delta_{I}^{m+r}(Y ; b)\right) \cdot \sum_{\substack{\max H \leq r \\
|H|=r(m+r-1) \\
I^{c} \subset \operatorname{Top} H}} Z^{H}+ \\
& +\left(\rho^{m+r}\right)^{*}\left(\pi_{I}^{*} \delta_{Y, *}^{|I|}\left(c_{1}(\mathscr{F})\right) \times \pi_{I^{c}}^{*}(\underbrace{b \times \ldots \times b}_{\left|I^{c}\right|})\right) \cdot \sum_{\substack{\max _{|H| r} \leq r(m+r-1)-1 \\
I^{c} \subset \operatorname{Top} H}}\left(\lambda_{H}(1)-1\right) Z^{H}+ \\
& +\left(\rho^{m+r}\right)^{*}\left(\pi_{I}^{*} \delta_{Y, *}^{|I|}\left(c_{1}(\mathscr{F})^{2}\right) \times \pi_{I^{c}}^{*}(\underbrace{b \times \ldots \times b}_{\left|I^{c}\right|})\right) \cdot \sum_{\substack{\max H \leq r \\
|H|=r(m+r-1)-2 \\
I^{c} \subset \operatorname{Top} H}} \frac{1}{2}\left(\lambda_{H}(1)-1\right)\left(\lambda_{H}(1)-2\right) Z^{H}+ \\
& +\left(\rho^{m+r}\right)^{*}\left(\pi_{I}^{*} \delta_{Y, *}^{|I|}\left(c_{2}(\mathscr{F})\right) \times \pi_{I^{c}}^{*}(\underbrace{b \times \ldots \times b}_{\left|I^{c}\right|})\right) \cdot \sum_{\substack{\max H \leq r \\
|H|=r(n-1)-2 \\
I^{c} \subset \operatorname{Top} H}}\left(\lambda_{H}(2)-1\right) Z^{H}+\mathscr{R}
\end{aligned}
$$

where

$$
\mathscr{R}=\sum_{\substack{\max H \leq r \\|H|<r(n-1)-2}} Q_{H} Z^{H}
$$


and each $Q_{H}$ appearing in (3.2.2) vanishes if the Chern classes of $\mathscr{F}$ of degree higher than 2 are zero. It follows that

$$
\begin{aligned}
& \Gamma^{m+r}(X ; a)=\sum_{\substack{\max H \leq r \\
|H|=r(m+r-1)}}\left(\rho^{m+r}\right)^{*}\left(\sum_{I^{c} \subset \operatorname{Top} H}(-1)^{m+r-|I|} \Delta_{I}^{m+r}(Y ; b)\right) \cdot Z^{H}+ \\
& \left.+\sum_{\substack{|+\max H \leq r\\
| H \mid=r(m+r-1)-1}}\left(\rho^{m+r}\right)^{*}\left(\sum_{I^{c} \subset \operatorname{Top} H}(-1)^{m+r-|I|}(\pi_{I}^{*} \delta_{Y, *}^{I I \mid}\left(c_{1}(\mathscr{F})\right) \times \pi_{I^{c}}^{*} \underbrace{b \times \ldots \times b}_{\left|I^{c}\right|})\right)\right) \cdot \epsilon_{H} Z^{H}+ \\
& \left.+\sum_{\substack{|H|=r(m+r-1)-2 \\
\mid \max _{\leq} \leq r}}\left(\rho^{m+r}\right)^{*}\left(\sum_{I^{c} \subset \operatorname{Top} H}(-1)^{m+r-|I|}(\pi_{I}^{*} \delta_{Y, *}^{|I|}\left(c_{1}(\mathscr{F})^{2}\right) \times \pi_{I^{c}}^{*} \underbrace{b \times \ldots \times b}_{\left|I^{c}\right|})\right)\right) \cdot \mu_{H} Z^{H}+ \\
& +\sum_{\substack{\max H \leq r \\
|H|=r(m+r-1)-2}}\left(\rho^{m+r}\right)^{*}\left(\sum_{I^{c} \subset \operatorname{Top} H}(-1)^{m+r-|I|}\left(\pi_{I}^{*} \delta_{Y, *}^{|I|}\left(c_{2}(\mathscr{F})\right) \times \pi_{I^{c}}^{*}(\underbrace{b \times \ldots \times b}_{\left|I^{c}\right|})\right) \cdot \nu_{H} Z^{H}+\mathscr{T}\right.
\end{aligned}
$$

where $\epsilon_{H}:=\left(\lambda_{H}(1)-1\right), \mu_{H}:=\left(\lambda_{H}(1)-1\right)\left(\lambda_{H}(1)-2\right) / 2, \nu_{H}:=\left(\lambda_{H}(2)-1\right)$, and $\mathscr{T}$ has an expansion similar to that of $\mathscr{R}$, see (3.2.2) and the comment following it.

Remark 3.6. Suppose that $\Gamma^{m}(Y ; b)=0$. Then the first addend on the right-hand side of (3.2.3) vanishes. In fact it is clearly independent of the rank- $r$ locally-free sheaf $\mathscr{F}$ and it is 0 for trivial $\mathscr{F}$ by Proposition $\mathbf{0 . 2}$ it follows that it vanishes.

3.3. $\mathbb{P}^{r}$-bundles over curves. We will prove Proposition 3.1 We start with an auxiliary result.

Claim 3.7. Let $Y$ be a smooth projective variety and $b \in Y$. Suppose that $\Gamma^{m}(Y ; b)=0$. Let $\mathfrak{z} \in \mathrm{CH}(Y)$ : then

$$
\sum_{I \subset\{1, \ldots,(m-1)\}}(-1)^{|I|} \pi_{I}^{*} \delta_{Y, *}^{|I|}(\mathfrak{z}) \times \pi_{I^{c}}^{*}(\underbrace{b, \ldots, b}_{\left|I^{c}\right|})=0 .
$$

Proof. Let $\pi_{\{1, \ldots,(m-1)\}}: Y^{m} \rightarrow Y^{m-1}$ be the projection to the first $(m-1)$ coordinates. Then

$$
\pi_{\{1, \ldots,(m-1)\}, *}\left(\Gamma^{m}(Y ; b) \cdot \pi_{m \mathfrak{z}}^{*}\right)=0 .
$$

The claim follows because the left-hand side of (3.3.2) equals the left-hand side of (3.3.1) multiplied by $(-1)^{m}$.

By (3.2.3) and Remark 3.6 we must prove that if $H=\left(h_{1}, \ldots, h_{m+r}\right)$ is a multi-index such that $\max H \leq r$ and $|H|=r(m+r-1)-1$ then

$$
\sum_{I^{c} \subset \operatorname{Top} H}(-1)^{m+r-|I|} \pi_{I}^{*} \delta_{Y, *}^{|I|}\left(c_{1}(\mathscr{F})\right) \times \pi_{I^{c}}^{*}(\underbrace{b, \ldots, b}_{\left|I^{c}\right|})=0 .
$$

A straightforward computation shows that $|\operatorname{Top} H| \geq(m-1)$ : thus (3.3.3) holds by Claim 3.7

3.4. $\mathbb{P}^{r}$-bundles over surfaces. We will prove Proposition $\mathbf{3 . 2}$ Notice that $\Gamma^{m}(Y ; b)=0$ : in fact it holds either by hypothesis or by Proposition 2.4 if $\Gamma^{m-1}(Y ; b)=0$. Moreover (3.3.3) holds in this case as well, the argument is that given in Subsection 3.3. Thus (3.2.3) and Remark $\mathbf{3 . 6}$ give that we must prove the following: if $H=\left(h_{1}, \ldots, h_{m+r}\right)$ is a multi-index such that $\max H \leq r$ and $|H|=r(m+r-1)-2$ then

$$
\sum_{I^{c} \subset \operatorname{Top} H}(-1)^{m+r-|I|}\left(\pi_{I}^{*} \delta_{Y, *}^{|I|}\left(\mu_{H} c_{1}(\mathscr{F})^{2}+\nu_{H} c_{2}(\mathscr{F})\right) \times \pi_{I^{c}}^{*}(\underbrace{b, \ldots, b}_{\left|I^{c}\right|})\right)=0 .
$$

A straightforward computation shows that $|\operatorname{Top} H| \geq(m-2)$ and that equality holds if and only if $(r-1) \leq h_{i} \leq r$ for all $1 \leq i \leq(m+r)$ (and thus the set of indices $i$ such that $h_{i}=(r-1)$ has cardinality $(r+2))$. If $\Gamma^{m-1}(Y ; b)=0$ then (3.4.1) holds by Claim 3.7. If both $c_{1}(\mathscr{F})^{2}, c_{2}(\mathscr{F})$ are multiples of $b$ then each term in the summation in the left-hand side of (3.4.1) is a multiple of $b$ and the coefficients sum up to 0 . 
3.5. Symmetric products of curves. If the genus of $C$ is 0 then $C^{(d)} \cong \mathbb{P}^{d}$ and hence the result holds trivially, see Example 1.2 Suppose that the genus of $C$ is 1 . If $d=1$ then $\Gamma^{3}(C ; p) \equiv 0$ by 6 . Let $d>1$ and let $u_{d}: C^{(d)} \rightarrow \operatorname{Pic}^{0}(C)$ be the map sending $D$ to $[D-d p]$. Since $u_{d}$ is $\mathbb{P}^{d-1}$-fibration we get that $\Gamma^{d+2}(C ; d p) \equiv 0$ by Proposition 3.3 and the equivalence $\Gamma^{3}(C ; p) \equiv 0$. Lastly suppose that the genus of $C$ is 2 . If $d=1$ then $\Gamma^{3}(C ; p) \equiv 0$ by [6] and if $d=2$ then $\Gamma^{5}\left(C^{(2)} ; 2 p\right) \equiv 0$ by Proposition 2.5. Now assume that $d>2$ and let $u_{d}: C^{(d)} \rightarrow \operatorname{Pic}^{0}(C)$ be the map sending $D$ to $[D-d p]$. Then $u_{d}$ is $\mathbb{P}^{d-2}$-fibration and we may write $C^{(d)} \cong \mathbb{P}\left(\mathscr{E}_{d}\right)$ where $\mathscr{E}_{d}$ is a locally-free sheaf on $\operatorname{Pic}^{0}(C)$ such that

$$
c_{1}\left(\mathscr{E}_{d}\right)=-[\{[x-p] \mid x \in C\}], \quad c_{2}\left(\mathscr{E}_{d}\right)=[\mathbf{0}],
$$

see Example 4.3 .3 of [5]. By Proposition 2.5 we have $\Gamma^{5}(J(C) ; \mathbf{0}) \equiv 0$; since $c_{1}\left(\mathscr{E}_{d}\right)^{2}=2[\mathbf{0}]$ we get that $\Gamma^{d+2}\left(C^{(d)} ; d p\right) \equiv 0$ by Proposition $\mathbf{3 . 3}$.

\section{BLOW-UPS}

We will prove Proposition $\mathbf{0 . 3}$. A comment regarding the hypotheses of Proposition $\mathbf{0 . 3}$. Let $Y$ be a complex $K 3$ surface and $X \rightarrow Y$ be the blow-up of $y \in Y$. We know (Beauville and Voisin) that there exists $c \in Y$ such that $\Gamma^{3}(Y ; c) \equiv 0$, but if $y$ is not rationally equivalent to $c$ then there exists no $a \in X$ such that $\Gamma^{3}(X ; a) \equiv 0$, this follows from Remark 1.1. If $e=0,1$ then Proposition 0.3 is trivial, hence we will assume that $e \geq 2$. We let $f: X \rightarrow Y$ be the blow-up of $V$ and $E \subset X$ the exceptional divisor of $f$. Thus $a \in E$. Let $g: E \rightarrow V$ be defined by the restriction of $f$ to $E$, and $(E / V)^{t}$ be the $t$-th fibered product of $g: E \rightarrow V$. Let $(E / V)^{t}$ be the $t$-th fibered product of $g: E \rightarrow V$. The following commutative diagram will play a rôle in the proof of Proposition $\mathbf{0 . 3}$

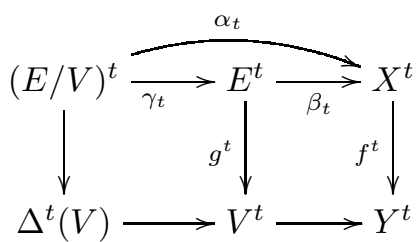

(The maps which haven't been defined are the natural ones.) Whenever there is no danger of confusion we denote $\alpha_{t}\left((E / V)^{t}\right)$ by $(E / V)^{t}$.

4.1. Pull-back of the modified diagonal. On $E$ we have an exact sequence of locally-free sheaves:

$$
0 \longrightarrow \mathscr{O}_{E}(-1) \longrightarrow g^{*} N_{V / Y} \longrightarrow Q \longrightarrow 0
$$

For $i=1, \ldots, t$ let $Q_{i}(t)$ be the pull-back of $Q$ to $E^{t}$ via the $i$-th projection $E^{t} \rightarrow E$ : thus $Q_{i}(t)$ is locally-free of rank $(e-1)$.

Proposition 4.1. Keep notation as above and let $d(t):=(t-1)(e-1)-1$. We have the following equalities in $\mathrm{CH}_{\operatorname{dim} X}\left(X^{t}\right)$ :

$$
\left(f^{t}\right)^{*} \Delta^{t}(Y)= \begin{cases}\Delta^{t}(X) & \text { if } t=1, \\ \Delta^{t}(X)+\beta_{t, *}\left(\left(g^{t}\right)^{*}\left(\Delta^{t}(V)\right) \cdot c_{d(t)}\left(\oplus_{j=1}^{t} Q_{j}(t)\right)\right) & \text { if } t>1 .\end{cases}
$$

Proof. The equality of schemes $f^{-1} \Delta^{1}(Y)=\Delta^{1}(X)$ gives (4.1.2) for $t=1$. Now let's assume that $t>1$. The closed set $\left(f^{t}\right)^{-1} \Delta^{t}(Y)$ has the following decomposition into irreducible components:

$$
\left(f^{t}\right)^{-1} \Delta^{t}(Y)=\Delta^{t}(X) \cup(E / V)^{t} .
$$

The dimension of $(E / V)^{t}$ is equal to $(\operatorname{dim} X+(t-1)(e-1)-1)$ and hence is larger than the expected dimension unless unless $2=t=e$. It follows that if $t=2$ and $e=2$ then $\left(f^{2}\right)^{*} \Delta^{2}(Y)=a \Delta^{2}(X)+$ $b(E / V)^{2}$ : one checks easily that $1=a=b$ and hence (4.1.2) holds if $t=2$ and $e=2$. Now suppose that that $t>1$ and $(t, e) \neq(2,2)$. Let $U:=\left(X^{t} \backslash\left(\Delta^{t}(X) \cap(E / V)^{t}\right)\right)$ and $\mathscr{Z}:=(E / V)^{t} \cap U=(E / V)^{t} \backslash \Delta^{t}(X)$. Notice that $(E / V)^{t}$ is smooth and hence the open subset $\mathscr{Z}$ is smooth as well. Let $\iota: \mathscr{Z} \hookrightarrow U$ be the inclusion. The restriction of $\left(f^{t}\right)^{*} \Delta^{t}(Y)$ to $U$ is equal to

$$
\left[\Delta^{t}(X) \cap U\right]+\iota_{*}\left(c_{d}(t)(\mathscr{N})\right)
$$

where $\mathscr{N}$ is the obstruction bundle (see [5], Cor. 8.1.2 and Prop. 6.1(a)). One easily identifies $\mathscr{N}$ with the restriction of $\oplus_{j=1}^{t} Q_{j}(t)$ to $\mathscr{Z}$. It follows that the restrictions to $U$ of the left and right hand sides of (4.1.2) are equal. The proposition follows because the dimension of $\left(X^{t} \backslash U\right)=\Delta^{t}(X) \cap(E / V)^{t}$ is equal to $(\operatorname{dim} X-1)$, which is strictly smaller than $\operatorname{dim} X$. 
Corollary 4.2. Keep notation and assumptions as above. Let $I \subset\{1, \ldots,(n+1)\}$ be non-empty and $I^{c}:=(\{1, \ldots,(n+1)\} \backslash I)$. Let $Q_{j}$ denote $Q_{j}(n+1)$ and let $t:=|I|$. Then

$$
\left(f^{n+1}\right)^{*} \Delta_{I}(Y ; b)= \begin{cases}\Delta_{I}(X ; a) & \text { if }|I|=1, \\ \Delta_{I}(X ; a)+\beta_{n+1, *}\left(\left(g^{n+1}\right)^{*} \Delta_{I}(V ; b) \cdot c_{d(t)}\left(\bigoplus_{j \in I} Q_{j}\right) \cdot \prod_{j \in I^{c}} c_{e-1}\left(Q_{j}\right)\right) & \text { if }|I|>1 .\end{cases}
$$

Proof. For $1 \leq i \leq(n+1)$ let $\rho_{i}: X^{n+1} \rightarrow X$ be the $i$-th projection. Let $J=\left\{j_{1}, \ldots, j_{m}\right\}$ where $1 \leq j_{1}<\ldots<j_{t} \leq(n+1)$, in particular $t=|J|$. We let $\pi_{J}: X^{n+1} \rightarrow X^{t}$ be the map such that the composition of the $i$-th projection $X^{t} \rightarrow X$ with $\pi_{J}$ is equal to $\rho_{j_{i}}$. The two maps $\pi_{I}: X^{n+1} \rightarrow X^{t}$ and $\pi_{I^{c}}: X^{n+1} \rightarrow X^{n+1-t}$ define an isomorphism $\Lambda_{I}: X^{n+1} \stackrel{\sim}{\longrightarrow} X^{t} \times X^{n+1-t}$. We have

$$
\left(f^{n+1}\right)^{*} \Delta_{I}(Y ; b)=\Lambda_{I}^{*}\left(\left(f^{t}\right)^{*} \Delta^{t}(Y) \times\left(f^{n+1-t}\right)^{*}(\{\underbrace{(b, \ldots, b)}_{n+1-t}\})\right) .
$$

(Here $\times$ denotes the exterior product of cycles, see 1.10 of [5.) An obstruction bundle computation gives that

$$
\left(f^{n+1-t}\right)^{*}(\{\underbrace{(b, \ldots, b)}_{n+1-t}\})=\beta_{n+1-t, *}\left(\prod_{1 \leq j \leq(n+1-t)} c_{e-1}\left(Q_{j}(n+1-t)\right)\right.
$$

The corollary follows from the above equations and Proposition 4.1

Let $I \subset\{1, \ldots,(n+1)\}$ be non-empty and let $t:=|I|$. We let $\Omega_{I} \in \mathrm{CH}_{\operatorname{dim} X}\left(E^{n+1}\right)$ be given by

$$
\Omega_{I}:= \begin{cases}0 & \text { if }|I|=1, \\ \left(g^{n+1}\right)^{*} \Delta_{I}(V ; b) \cdot c_{d(t)}\left(\bigoplus_{j \in I} Q_{j}\right) \cdot \Pi_{j \in I^{c}} c_{e-1}\left(Q_{j}\right) & \text { if }|I|>1 .\end{cases}
$$

By Corollary 4.2 we have $\left(f^{n+1}\right)^{*} \Delta_{I}(Y ; b)=\Delta_{I}(X ; a)+\beta_{n+1, *}\left(\Omega_{I}\right)$ and hence

$$
\left(f^{n+1}\right)^{*}\left(\Gamma^{n+1}(Y ; b)\right)=\Gamma^{n+1}(X ; a)+\beta_{n+1, *}\left(\sum_{1 \leq|I| \leq(n+1)}(-1)^{n+1-|I|} \Omega_{I}\right) .
$$

4.2. The proof. By (4.1.9) it suffices to prove that the following equality holds in $\mathrm{CH}_{\operatorname{dim} X}\left(E^{n+1}\right)_{\mathbb{Q}}$ :

$$
\sum_{1 \leq|I| \leq(n+1)}(-1)^{|I|} \Omega_{I}=0 .
$$

Let $I \subset\{1, \ldots,(n+1)\}$ be of cardinality strictly greater than $(n-e)$ : Corollary $\mathbf{2 . 2}$ allows us to express the class of $\Delta_{I}(V ; b)$ as a linear combination of the $\Delta_{J}(V ; b)$ 's with $J \subset I$ of cardinality at most $(n-e)$. Moreover Whitney's formula allows us to write the Chern class appearing in the definition of $\Omega_{I}$ as a sum of products of Chern classes of the $Q_{j}$ 's. It follows that for each $I \subset\{1, \ldots,(n+1)\}$ we may express the class of $\Omega_{I}$ as a linear combination of the classes

$$
\left(g^{n+1}\right)^{*} \Delta_{J}(V ; b) \cdot \prod_{s=1}^{n+1} c_{k_{s}}\left(Q_{s}\right), \quad 1 \leq|J| \leq(n-e), \quad k_{1}+\ldots+k_{n+1}=d(n+1)=n(e-1)-1 .
$$

Definition 4.3. $\mathscr{P}_{n}(e)$ is the set of $(n+1)$-tuples $k_{1}, \ldots, k_{n+1}$ of natural numbers $0 \leq k_{s} \leq(e-1)$ whose sum equals $d(n+1)$.

Summing over all $I \subset\{1, \ldots,(n+1)\}$ of a given cardinality $t$ we get the following.

Claim 4.4. Let $1 \leq t \leq(n+1)$. There exists an integer $c_{J, K}(t)$ for each couple $(J, K)$ with $\emptyset \neq J \subset$ $\{1, \ldots,(n+1)\}$ of cardinality at most $(n-e)$ and $K \in \mathscr{P}_{n}(e)$ such that

$$
\sum_{|I|=t} \Omega_{I}=\sum_{\substack{1 \leq|J| \leq(n-e) \\ K \in \mathscr{P}_{n}(e)}} c_{J, K}(t)\left(g^{n+1}\right)^{*} \Delta_{J}(V ; b) \cdot \prod_{s=1}^{n+1} c_{k_{s}}\left(Q_{s}\right) .
$$

It will be convenient to set $c_{J, K}(0)=0$. We will prove that

$$
\sum_{t=0}^{n+1}(-1)^{t} c_{J, K}(t)=0
$$

That will prove Equation (4.2.1) and hence also Proposition $\mathbf{0 . 3}$. Applying Corollary $\mathbf{2 . 2}$ to $(V, b)$ we get the following result. 
Claim 4.5. Let $I \subset\{1, \ldots, n+1\}$ be of cardinality $t \geq(n+1-e)$. Then

$$
\Delta_{I}^{n+1}(V ; b) \equiv \sum_{\substack{J \subset I \\
1 \leq|J| \leq(n-e)}}(-1)^{n-e-|J|}\left(\begin{array}{c}
t-|J|-1 \\
t-n-1+e
\end{array}\right) \Delta_{J}^{n+1}(Y ; b) .
$$

Given $K \in \mathscr{P}_{n}(e)$ we let

$$
T(K):=\left\{1 \leq i \leq(n+1) \mid k_{i}=(e-1)\right\} .
$$

A simple computation gives that

$$
(n+1-e) \leq|T(K)|
$$

Proposition 4.6. Let $\emptyset \neq J \subset\{1, \ldots,(n+1)\}$ be of cardinality at most $(n-e)$, let $K \in \mathscr{P}_{n}(e)$ and $0 \leq t \leq(n+1)$. Then

$$
c_{J, K}(t)=(-1)^{n-|J|-e}\left(\begin{array}{c}
t-|J|-1 \\
n-|J|-e
\end{array}\right)\left(\begin{array}{c}
\left|T(K) \cap J^{c}\right| \\
n+1-t
\end{array}\right)
$$

Proof. Suppose first that $0 \leq t \leq(n-e)$. Then $c_{J, K}(t)=0$ unless $|J|=t$ and $J^{c} \subset T(K)$ : if the latter holds then $c_{J, K}(t)=1$. Assume that the right-hand side of (4.2.8) is non-zero: then the first binomal coefficient is non-zero and hence $t \leq|J|$. Of course also the second binomal coefficient is non-zero: it follows that

$$
(n+1-t) \leq\left|T(K) \cap J^{c}\right| \leq\left|J^{c}\right|=n+1-|J| .
$$

Since $t \leq|J|$ it follows that $|J|=t$ and hence $\left|T(K) \cap J^{c}\right|=\left|J^{c}\right|$ i.e. $J^{c} \subset T(K)$ : a straightforward computation gives that under these assumptions the right-hand side of (4.2.8) equals 1 . It remains to prove that (4.2.8) holds for $(n+1-e) \leq t \leq(n+1)$. Looking at (4.1.8) and Claim 4.5 we get that

$$
c_{J, K}(t)=(-1)^{n-e-|J|}\left(\begin{array}{c}
t-|J|-1 \\
t-n-1+e
\end{array}\right)\left|\left\{I \subset\{1, \ldots,(n+1)\}\left|I^{c} \subset(T(K) \cap J), \quad\right| I \mid=t\right\}\right| .
$$

Since the right-hand side of (4.2.10) is equal to the right-hand side of (4.2.8) this finishes the proof.

Let

$$
p(x):=\left(\begin{array}{c}
n-|J|-x \\
n-|J|-e
\end{array}\right) .
$$

Then $\operatorname{deg} p<\left|T(K) \cap J^{c}\right|$ because $\operatorname{deg} p=(n-|J|-e)$ and because (4.2.77) gives that

$$
\left|T(K) \cap J^{c}\right| \geq(n+1-e)+(n+1-|J|)-(n+1)=n-|J|-e+1 .
$$

Thus (2.2.1) and (4.2.8) give that

$$
0=\sum_{s=0}^{n+1}(-1)^{s} p(s)\left({ }^{\left|T(K) \cap J^{c}\right|}\right)=(-1)^{n+1} \sum_{t=0}^{n+1}(-1)^{t}\left(\begin{array}{c}
t-|J|-1 \\
n-|J|-e
\end{array}\right)\left(\begin{array}{c}
\left|T(K) \cap J^{c}\right| \\
n+1-t
\end{array}\right)=(-1)^{1-e-|J|} \sum_{t=0}^{n+1}(-1)^{t} c_{J, K}(t) .
$$

This finishes the prooof of Proposition 0.3 .

4.3. Application to Hilbert schemes of $K 3$ 's. Let $S$ be a complex $K 3$ surface. By Beauville and Voisin [3] there exists $c \in S$ such that $\Gamma^{3}(S ; c) \equiv 0$. We let $S^{[n]}$ be the Hilbert scheme parametrizing length- $n$ subschemes of $S$; Beauville [1] proved that $S^{[n]}$ is a hyperkähler variety.

Proposition 4.7. Keep notation as above and assume that $n=2,3$. Let $a_{n} \in S^{[n]}$ represent a scheme supported at $c$. Then $\Gamma^{2 n+1}\left(S^{[n]} ; a_{n}\right) \equiv 0$.

Proof. First assume that $n=2$. Let $\pi_{1}: X \rightarrow S \times S$ be the blow-up of the diagonal $\Delta$ and $\rho_{2}: X \rightarrow S^{(2)}$ the composition of $\pi_{1}$ and the quotient map $S \times S \rightarrow S^{(2)}$. There is a degree-2 map $\phi_{2}: X \rightarrow S^{[2]}$ fitting into a commutative diagram

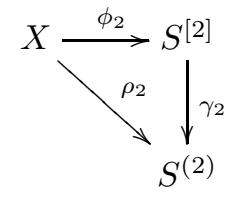

where $\gamma_{2}([Z])=\sum_{p \in S} \ell\left(\mathscr{O}_{Z}, p\right)$ is the Hilbert-Chow morphism. Let $x \in X$ such that $\phi_{2}(x)=a_{2}$; by Subsection 1.3 it suffices to prove that $\Gamma^{5}(X ; x) \equiv 0$. By commutativity of (4.3.14) we have $\pi_{1}(x)=(c, c)$. Now $\Gamma^{5}(S \times S ;(c, c)) \equiv 0$ by Proposition 0.2 and since $\operatorname{cod}(\Delta, S \times S)=2$ it follows from Proposition $\mathbf{0 . 3}$ that $\Gamma^{5}(X ; x) \equiv 0$. Next assume that $n=3$. Let $\pi_{2}: Y \rightarrow S^{[2]} \times S$ be the blow-up with center the tautological subscheme $\mathscr{Z}_{2} \subset S^{[2]} \times S$ and $\rho_{3}: Y \rightarrow S^{(3)}$ the composition of $\pi_{2}$ 
and the natural map $S^{[2]} \times S \rightarrow S^{(3)}$. There is a degree-3 map $\phi_{3}: Y \rightarrow S^{[3]}$ fitting into a commutative diagram

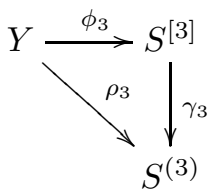

where $\gamma_{3}$ is the Hilbert-Chow morphism. (See for example Proposition 2.2 of [4.) On the other hand let $p_{1}: S \times S \rightarrow S$ be projection to the first factor; the map

$$
\left.\left(\phi_{2}, p_{1} \circ \pi_{1}\right)\right): X \rightarrow S^{[2]} \times S
$$

is an isomorphism onto $\mathscr{Z}_{2}$. Let $y \in Y$ be such that $\phi_{3}(y)=a_{3}$; by Subsection 1.3 it suffices to prove that $\Gamma^{7}(Y ; y) \equiv 0$. Notice that $\pi_{2}(y)=\left(a_{2}, c\right)$ where $a_{2} \in S^{[2]}$ is supported at $c$. By the case $n=2$ (that we just proved) and Proposition 0.2 we have $\Gamma^{7}\left(S^{[2]} \times S ;\left(a_{2}, c\right)\right) \equiv 0$. Let $x \in X$ such that $\phi_{2}(x)=a_{2}$. In the proof for the case $n=2$ we showed that $\Gamma^{5}(X ; x) \equiv 0$; since (4.3.16) is an isomorphism it follows that $\Gamma^{5}\left(\mathscr{Z}_{2} ;\left(a_{2}, c\right)\right) \equiv 0$. Since $\Gamma^{7}\left(S^{[2]} \times S ;\left(a_{2}, c\right)\right) \equiv 0$ and $\mathscr{Z}_{2}$ is smooth of codimension 2 , we get $\Gamma^{7}(Y ; y) \equiv 0$ by Proposition $\mathbf{0 . 3}$.

Let $\mathscr{Z}_{n} \subset S^{[n]} \times S$ be the tautological subscheme. The blow-up of $S^{[n]} \times S$ with center $\mathscr{Z}_{n}$ has a natural regular map of finite (non-zero) degree to $S^{[n+1]}$ and in turn $\mathscr{Z}_{n}$ may be described starting from the tautological subscheme $\mathscr{Z}_{n-1} \subset S^{[n-1]} \times S$. Thus one may hope to prove by induction on $n$ that $\Gamma^{2 n+1}\left(S^{[n]} ; a\right) \equiv 0$ for any $n$ : the problem is that starting with $\mathscr{Z}_{3}$ the tautological subscheme is singular.

\section{Double COvers}

In the present section we will assume that $X$ is a projective variety over a field $\mathbb{K}$ and that $\iota \in \operatorname{Aut}(X)$ is a (non-trivial) involution. We let $Y:=X /\langle\iota\rangle$ and $f: X \rightarrow Y$ be the quotient map. We assume that there exists $a \in X(\mathbb{K})$ which is fixed by $\iota$ and we let $b:=f(a)$.

Conjecture 5.1. Keep hypotheses and notation as above and suppose that $\Gamma^{m}(Y ; b) \equiv 0$. Then $\Gamma^{2 m-1}(X ; a) \equiv 0$.

The above conjecture was proved for $m=2$ by Gross and Schoen, see Prop. 4.8 of $[6$. We will propose a proof of Conjecture $\mathbf{5 . 1}$ and we will show that the proof works for $m=2,3$. Of course the proof for $m=2$ is that of Gross and Schoen (with the symmetric cube of the curve replaced by the cartesian cube).

5.1. A modest proposal. There is a well-defined pull-back homomorphisms

$$
\left(f^{q}\right)^{*}: Z_{*}\left(Y^{q}\right)_{\mathbb{Q}} \rightarrow Z_{*}\left(X^{q}\right)_{\mathbb{Q}}
$$

compatible with rational equivalence (see Ex. 1.7.6 of [5]): thus we have an induced homomorphism $\left(f^{q}\right)^{*}: \mathrm{CH}_{*}\left(Y^{q}\right)_{\mathbb{Q}} \rightarrow \mathrm{CH}_{*}\left(X^{q}\right)_{\mathbb{Q}}$. Let $n:=\operatorname{dim} X$ and $\Xi_{m} \in Z_{n}\left(X^{m}\right)_{\mathbb{Q}}$ the cycle defined by

$$
\Xi_{m}:=\left(f^{m}\right)^{*} \Gamma^{m}(Y ; b) .
$$

We will show that $\Xi_{m}$ is a linear combination of cycles of the type

$$
\{(x, \ldots, \iota(x), \ldots x, \ldots, x, a, \ldots \iota(x), \ldots, a, \ldots) \mid x \in X\} .
$$

Notice that the $\Delta_{I}(X ; a)$ 's are of this type. Consider the inclusions of $X^{m}$ in $X^{2 m-1}$ which map $\left(x_{1}, \ldots, x_{m}\right)$ to $\left(x_{1}, \ldots, x_{m}, \nu(1), \ldots, \nu(m-1)\right)$ where $\nu:\{1, \ldots,(m-1)\} \rightarrow\left\{a, x_{1}, \ldots, x_{m}, \iota\left(x_{1}\right), \ldots, \iota\left(x_{m}\right)\right\}$ is an arbitrary list. Let $\Phi_{\nu}\left(\Xi_{m}\right)$ be the symmetrized image of $\Xi_{m}$ in $Z_{n}\left(X^{2 m-1}\right)$ for the inclusion determined by $\nu$ : it is a linear combination of cycles (5.1.3). By hypothesis $\Xi_{m} \equiv 0$ and hence any linear combination of the cycles $\Phi_{\nu}\left(\Xi_{m}\right)$ is rationally equivalent to 0 . One gets the proof if a suitable linear combination of the $\Phi_{\nu}\left(\Xi_{m}\right)$ 's is a linear combination of the $\Delta_{I}(X ; a)$ 's with the appropriate coefficients (so that it is equal to a non-zero multiple of $\Gamma^{2 m-1}(X ; a)$ ). We will carry out the proof for $m=2,3$. 
5.2. Preliminaries. Since the involution of $X$ is non-trivial the dimension of $X$ is strictly positive i.e. $n>0$. Let $\mu:\{1, \ldots, q\} \rightarrow\{a, x, \iota(x)\}$. If $\mu$ is not the sequence $\mu(1)=\ldots=\mu(q)=a$ we let

$$
\Omega(\mu(1), \ldots, \mu(q)):=\left\{\left(x_{1}, \ldots, x_{q}\right) \in X^{q} \mid x_{i}=\mu(i), \quad x \in X\right\},
$$

and we let $\Omega(a, \ldots, a):=0$. Thus $\Omega(\mu(1), \ldots, \mu(d))$ is an $n$-cycle on $X^{d}$. For example $\Omega(x, \ldots, x) \in X^{q}$ is the small diagonal. Let $\mathscr{S}_{q}$ be the symmetric group on $\{1, \ldots, q\}$ : of course it acts on $X^{q}$. For $r+s+t=q$ let

$$
\bar{\Omega}(r, s, t):=\sum_{\sigma \in \mathscr{S}_{q}} \sigma(\Omega(\underbrace{a, \ldots, a}_{r}, \underbrace{x, \ldots, x}_{s}, \underbrace{\iota(x), \ldots, \iota(x)}_{t})) .
$$

Thus $\bar{\Omega}(r, s, t)$ is an $n$-cycle on $X^{q}$ invariant under the action of $\mathscr{S}_{q}$. Notice that

$$
\bar{\Omega}(r, s, t)=\bar{\Omega}(r, t, s) .
$$

With this notation

$$
\Gamma^{q}(X ; a)=\sum_{\substack{0 \leq r, s \\ r+s=q}} \frac{(-1)^{r}}{r ! s !} \bar{\Omega}(r, s, 0) .
$$

Let $\Xi_{m}$ be the cycle on $X^{m}$ given by (5.1.2). A straightforward computation gives that

$$
2 \Xi_{m}=\sum_{\substack{0 \leq r, s, t \\ r+s+t=m}} \frac{(-2)^{r}}{r ! s ! t !} \bar{\Omega}(r, s, t) .
$$

(Equality (5.2.3) is the reason for the factor of 2 in front of $\Xi_{m}$.) For

$$
\nu:\{1, \ldots,(m-1)\} \rightarrow\left\{a, x_{1}, \ldots, x_{m}, \iota\left(x_{1}\right), \ldots, \iota\left(x_{m}\right)\right\}
$$

we let

$$
\begin{array}{ccc}
X^{m} & \stackrel{j_{\nu}}{\longmapsto} & X^{2 m-1} \\
\left(x_{1}, \ldots, x_{m}\right) & \mapsto & \left(x_{1}, \ldots, x_{m}, \nu(1), \ldots, \nu(m-1)\right)
\end{array}
$$

and $\Phi_{\nu}: Z_{n}\left(X^{m}\right) \rightarrow Z_{n}\left(X^{2 m-1}\right)$ be the homomorphism

$$
\Phi_{\nu}(\gamma):=\sum_{\sigma \in \mathscr{S}_{2 m-1}} \sigma_{*}\left(j_{\nu, *}(\gamma)\right) .
$$

Notice that $\Phi_{\nu}$ does not change if we reorder the sequence $\nu$.

5.3. The case $m=2$. A straightforward computation (recall (5.2.3)) gives that

$$
\begin{aligned}
\Phi_{a}\left(\Xi_{2}\right) & =\bar{\Omega}(1,2,0)-4 \bar{\Omega}(2,1,0)+\bar{\Omega}(1,1,1), \\
\Phi_{x_{1}}\left(\Xi_{2}\right) & =\bar{\Omega}(0,3,0)-2 \bar{\Omega}(1,2,0)-2 \bar{\Omega}(2,1,0)+\bar{\Omega}(0,2,1), \\
\Phi_{\iota\left(x_{1}\right)}\left(\Xi_{2}\right) & =-2 \bar{\Omega}(2,1,0)-2 \bar{\Omega}(1,1,1)+2 \bar{\Omega}(0,2,1) .
\end{aligned}
$$

Thus

$$
0 \equiv-2 \Phi_{a}\left(\Xi_{2}\right)+2 \Phi_{x_{1}}\left(\Xi_{2}\right)-\Phi_{\iota\left(x_{1}\right)}\left(\Xi_{2}\right)=2 \bar{\Omega}(0,3,0)-6 \bar{\Omega}(1,2,0)+6 \bar{\Omega}(2,1,0)=12 \Gamma^{3}(X ; a) .
$$

5.4. The case $m=3$. For every $\nu:\{1,2\} \rightarrow\left\{a, x_{1}, x_{2}, x_{3}, \iota\left(x_{1}\right), \iota\left(x_{2}\right), \iota\left(x_{3}\right)\right\}$ the cycle $\Phi_{\nu}\left(\Xi_{3}\right)$ is equal to the linear combination of the classes listed in the first column of Table (11) with coefficients the numbers in the corresponding column of Table (11). For such a $\nu$ let $i(\nu)$ be its position in the first row of Table (1): thus $i((a, a))=1, \ldots, i\left(\left(\iota\left(x_{1}\right), \iota\left(x_{2}\right)\right)=9\right.$. Table (1) allows us to rewrite

$$
\sum_{\nu} \lambda_{i(\nu)} \Phi_{\nu}\left(\Xi_{3}\right)
$$

as an integral linear combination of the classes listed in the first column of Table (1), with coefficients $F_{1}, \ldots, F_{9}$ which are linear functions of $\lambda_{1}, \ldots, \lambda_{9}$. Let's impose that $0=F_{1}=\ldots=F_{6}$ : solving the 
TABLE 1. Coordinates of $\Phi_{\nu}(\Xi)$ for $\nu=(a, a), \ldots,\left(\iota\left(x_{1}\right), \iota\left(x_{2}\right)\right)$.

\begin{tabular}{lccccccccc}
\hline & $(a, a)$ & $\left(a, x_{1}\right)$ & $\left(a, \iota\left(x_{1}\right)\right)$ & $\left(x_{1}, x_{1}\right)$ & $\left(x_{1}, x_{2}\right)$ & $\left(x_{1}, \iota\left(x_{1}\right)\right)$ & $\left(x_{1}, \iota\left(x_{2}\right)\right)$ & $\left(\iota\left(x_{1}\right), \iota\left(x_{1}\right)\right)$ & $\left(\iota\left(x_{1}\right), \iota\left(x_{2}\right)\right)$ \\
\hline $\bar{\Omega}(3,1,1)$ & -6 & -2 & 2 & -2 & 0 & -2 & 4 & -2 \\
\hline $\bar{\Omega}(2,2,1)$ & 3 & -4 & -8 & 0 & -4 & 4 & -6 & -4 & -4 \\
\hline $\bar{\Omega}(1,3,1)$ & 0 & 2 & 2 & -4 & 0 & -4 & -4 & -4 \\
\hline $\bar{\Omega}(1,2,2)$ & 0 & 1 & 2 & 0 & -2 & 1 & 3 & 3 \\
\hline $\bar{\Omega}(0,4,1)$ & 0 & 0 & 0 & 2 & 1 & 3 & 0 \\
\hline $\bar{\Omega}(0,3,2)$ & 0 & 0 & 0 & 1 & 2 & 0 & 0 \\
\hline $\bar{\Omega}(0,5,0)$ & 0 & 0 & 0 & 1 & 1 & 0 & 0 & 0 \\
\hline $\bar{\Omega}(1,4,0)$ & 0 & 1 & 0 & -4 & -2 & 0 & 0 & 0 \\
\hline $\bar{\Omega}(2,3,0)$ & 1 & -4 & 0 & 4 & -4 & 0 & -4 & 0 \\
\hline $\bar{\Omega}(3,2,0)$ & -6 & 2 & -2 & -2 & 8 & -2 & 4 & 0 \\
\hline $\bar{\Omega}(4,1,0)$ & 12 & 8 & 8 & 8 & 4 & 8 & 4 & 0 \\
\hline
\end{tabular}

corresponding linear system we get that

$$
\begin{aligned}
& \lambda_{1}=\frac{1}{3}\left(-8 \lambda_{6}-2 \lambda_{7}-8 \lambda_{8}-8 \lambda_{9}\right), \\
& \lambda_{2}=\frac{1}{3}\left(14 \lambda_{6}+8 \lambda_{7}+14 \lambda_{8}+20 \lambda_{9}\right), \\
& \lambda_{3}=\frac{1}{3}\left(-6 \lambda_{6}-6 \lambda_{7}-6 \lambda_{8}-12 \lambda_{9}\right), \\
& \lambda_{4}=\frac{1}{3}\left(\lambda_{6}-2 \lambda_{7}+\lambda_{8}+4 \lambda_{9}\right), \\
& \lambda_{5}=\frac{1}{3}\left(-5 \lambda_{6}-2 \lambda_{7}-5 \lambda_{8}-8 \lambda_{9}\right) .
\end{aligned}
$$

For such a choice of coefficients $\lambda_{1}, \ldots, \lambda_{9}$ we have that

$0 \equiv \sum_{\nu} \lambda_{i(\nu)} \Phi_{\nu}(\Xi)=-\frac{4}{3}\left(\lambda_{6}+\lambda_{7}+\lambda_{8}+\lambda_{9}\right)(\bar{\Omega}(0,5,0)-5 \bar{\Omega}(1,4,0)+10 \bar{\Omega}(2,3,0)-10 \bar{\Omega}(3,2,0)+5 \bar{\Omega}(4,1,0))$.

Choosing integers $\lambda_{6}, \ldots, \lambda_{9}$ such that $\left(\lambda_{6}+\lambda_{7}+\lambda_{8}+\lambda_{9}\right)=-3$ we get that

$$
0 \equiv \sum_{\nu} \lambda_{i(\nu)} \Phi_{\nu}(\Xi)=4 \cdot 5 ! \Gamma^{5}(X ; a) .
$$

This concludes the proof of Conjecture 5.1 for $m=3$.

\section{REFERENCES}

1. A. Beauville, Variétes Kähleriennes dont la premiére classe de Chern est nulle, J. Differential geometry 18, 1983, pp. 755-782.

2. A. Beauville, On the splitting of the Bloch-Beilinson filtration, Algebraic cycles and motives. Vol. 2, LMS Lecture Note Ser. 344 (2007), pp. 38-53.

3. A. Beauville - C. Voisin, On the Chow ring of a K3 surface, J. Algebraic Geometry 13 (2004), pp. 417-426.

4. G. Ellingsrud - S. A. Stromme, An intersection number for the punctual Hilbert scheme of a surface, Trans.of the AMS 350 (1998), pp. 2547-2552.

5. W. Fulton, Intersection theory, Ergebnisse der Mathematik und ihrer Grenzgebiete (3) 2. Springer-Verlag, Berlin, 1984.

6. B. H. Gross - C. Schoen, The modified diagonal cycle on the triple product of a pointed curve, Ann. Inst. Fourier 45 (1995), pp. 649-679.

7. Lie Fu, Beauville-Voisin conjecture for generalized Kummer varieties, arXiv:1309.4977 [math.AG].

8. B. Moonen - Q. Yin, On a question of O'Grady about modified diagonals, arXiv:1311.1185] [math.AG].

9. Mingmin Shen - C. Vial, The Fouruer transform for certain hyperkähler fourfolds, arXiv:1309.5965 [math.AG].

10. C. Voisin, On the Chow ring of certain algebraic hyper-Kähler manifolds, Pure Appl. Math. Q. 4 (2008), pp. 613-649. 
11. C. Voisin, Infinitesimal invariants for cycles modulo algebraic equivalence and 1-cycles on Jacobians, arXiv:1304.4095 [math.AG]. 